\title{
Surface Expression of Bcl-2 in Chronic Lymphocytic Leukemia and Other B-Cell Leukemias and Lymphomas Without a Breakpoint $t(14 ; 18)$
}

\author{
Brian A McCarthy, ${ }^{1}$ Erin Boyle, ${ }^{1}$ Xue Ping Wang, ${ }^{2}$ Dorothy Guzowski, ${ }^{2}$ Santanu Paul, ${ }^{1}$ Rosa Catera, ${ }^{1}$ \\ Joshua Trott, ${ }_{1}$ Xiao-jie Yan, ${ }_{1}$ Carlo M Croce, ${ }^{3}$ Rajendra Damle, ${ }^{1}$ Sophia Yancopoulos, ${ }^{1}$ Bradley T Messmer, \\ Martin Lesser, ${ }^{5}$ Steven L Allen, ${ }^{1,5}$ Kanti R Rai, ${ }^{1,6}$ and Nicholas Chiorazzi ${ }^{1,5}$ \\ ${ }^{1}$ Laboratory of Experimental Immunology, ${ }^{2}$ Core Facility, The Feinstein Institute for Medical Research, Manhasset, New York, United \\ States of America; ${ }^{3}$ Ohio State University, Department of Molecular Virology, Immunology, and Medical Genetics and Comprehensive \\ Cancer Center, Columbus, Ohio, United States of America; ${ }^{4}$ Moores Cancer Center, University of California at San Diego, La Jolla, \\ California, United States of America; ${ }^{5}$ Department of Medicine, North Shore University Hospital, Manhasset, New York, United \\ States of America; and ${ }^{6}$ Department of Medicine, Long Island Jewish Medical Center, Manhasset, New York, United States of America
}

\begin{abstract}
Since its discovery in follicular lymphoma cells at the breakpoint $\mathrm{t}(14 ; 18), \mathrm{BCl}-2$ has been studied extensively in many basic and clinical science settings. Bcl-2 can locate as an integral mitochondrial membrane component, where its primary role is to block apoptosis by maintaining membrane integrity. Here we show that $\mathrm{Bcl}-2$ also can position on the outer cell surface membrane of B cells from patients with chronic lymphocytic leukemia (B-CLL) and certain other leukemias that do not classically possess the chromosomal breakpoint $\mathrm{t}(14 ; 18)$. Although low levels of $\mathrm{BCl}-2$ can be detected on the surface membrane of apparently healthy leukemic and normal B cells, expression of $\mathrm{BCl}-2$ correlates best with spontaneous or induced apoptosis. Notably, upon induction of apoptosis, B-CLL cells were much more efficient in upregulating surface Bcl-2 than normal B cells. It is not clear if this surface membrane expression is a passive consequence of the apoptotic process or an active attempt by the B cell to abort cell death by stabilizing the plasma membrane.
\end{abstract}

Online address: http://www.molmed.org

doi: $10.2119 / 2008.00061$. McCarthy

\section{INTRODUCTION}

Bcl-2 was identified initially at a common chromosomal breakpoint in B-cell follicular lymphomas $t(14 ; 18)(1)$. This seminal finding led to the discovery of a family of $\sim 20$ proteins involved in the regulation of programmed cell death (reviewed in [2]). The Bcl-2 family contains both anti- and pro-apoptotic members, and the balance of these counteracting forces is crucial to the fate and expansion of normal and neoplastic B lymphocytes. $\mathrm{Bcl}-2$ can play a primary role in oncogenesis by inhibiting apoptosis, and was the first member of a new category of oncogenes: regulators of cell death (3).

The anti-apoptotic members of the Bcl-2 family are involved in checkpoints upstream of mitochondrial dysfunction and caspase activation (3). By virtue of its pivotal position in the life and death cycle of a cell, Bcl-2 may be an excellent candidate for therapeutic intervention $(4,5)$. Early clinical trials utilizing an antisense strategy to alter Bcl-2 levels have shown some encouraging initial results (6); additional promising results were reported recently using small molecule in-
Address correspondence and reprint requests to Nicholas Chiorazzi, The Feinstein Institute for Medical Research, 350 Community Drive, Manhasset, NY 1 1030, USA. Phone: 516-5621090; Fax: 516-562-101 1; E-mail: NChizzi@NSHS.edu.

Submitted May 13, 2008; Accepted for publication July 7, 2008; Epub (WwW.molmed.org) ahead of print July 9, 2008. hibitors of Bcl-2 function, such as the BH3 mimetic ABT-737 (7).

After synthesis, Bcl-2 is chaperoned to several intracellular sites by FKBP38 (8), an FK506-binding immunophilin protein (9). At these sites, Bcl-2 becomes integrated into the cytoplasmic face of the intracellular membranes by its carboxyl-terminal, hydrophobic 17 amino acid tail (10). Bcl-2's role, when positioned at the mitochondrial surface, is to maintain organelle integrity and prevent apoptosis $(11,12)$. In addition, the molecule is associated with the cytoplasmic surfaces of the endoplasmic reticulum (ER) and the nuclear envelope (13). Paradoxically, when localized to the nuclear compartment, Bcl-2 can induce apoptosis by inhibiting transcription factor transport into the nucleus, thus highlighting that cellular sublocalization can determine opposing actions (14). 
In this manuscript, we demonstrate the presence of $\mathrm{Bcl}-2$ on the surface membrane of human B lymphocytes, especially leukemic B cells from patients with B-cell type chronic lymphocytic leukemia (B-CLL) and certain related diseases. Although the function of cell surface-associated Bcl-2 is not clear, its appearance, primarily on cells undergoing apoptosis, suggests a relationship between surface membrane re-localization and the apoptotic process.

\section{MATERIALS AND METHODS}

\section{Patients and Healthy Donors}

The Institutional Review Board of the North Shore-LIJ Health System approved these studies. Following informed consent obtained in accordance with the Declaration of Helsinki, peripheral venous blood was taken from B-CLL patients and healthy subjects. B-CLL clones expressing IGHV genes differing by $\geq 2 \%$ from the most similar germline gene were defined as Mutated-CLL (M-CLL), and clones expressing IGHV genes with $<2 \%$ difference from germline gene as UnmutatedCLL (U-CLL).

For gene expression profiling studies, peripheral blood mononuclear cells (PBMC) were separated by FicollHypaque (Ficoll-Paque; Pharmacia LKB Biotechnology, Piscataway, NJ, USA) density gradient centrifugation, and then $\mathrm{B}$ cells were isolated by negative selection using the B-cell Isolation Kit (Miltenyi, Auburn, CA, USA) and MScolumns. For real-time quantitative polymerase chain reaction (RT-QPCR) verification, freshly isolated B-cells from B-CLL patients and control subjects matched for age ( $\geq 55$ years) were separated by density gradient centrifugation and isolated by negative selection using RosetteSep (StemCell Technologies, Vancouver, Canada) according to the manufacturer's protocol. In some cases, particularly other malignancies, PBMC were cryopreserved with DMSO using a programmable cell-freezing machine (CryoMed, Mt. Clemens, MI, USA). These were subsequently thawed and analyzed.
In both cases, total RNA was isolated with the RNeasy kit (Qiagen, Valencia, CA, USA) as suggested by the manufacturer. For microarray analysis, double stranded cDNA was synthesized from total RNA using a T7-polyT primer and the SuperScript Choice System Kit (GibcoBRL/Invitrogen Carlsbad, CA, USA). cDNA was purified by phenol/ chloroform extraction and biotinylated by in vitro transcription using the Bio Array High Yield RNA transcript labeling kit (ENZO, Farmingdale, NY, USA). cRNA was purified and DNAse treated using the RNeasy kit, fragmented according to the Affymetrix protocol, and $15 \mu \mathrm{g}$ hybridized to the Human HG-U95 or Human HG-U133 microarray chip sets (Affymetrix, Santa Clara, CA, USA). Chip hybridization, washing, and imaging were performed by the Core Facility of The Feinstein Institute for Medical Research following Affymetrix protocols and analyzed using Affymetrix-provided software. We used .cel and .txt files thus generated for subsequent analyses with Microsoft Excel (Redmond, WA, USA), DChip (Boston, MA, USA), GeneCluster (Cambridge, MA, USA), and Genesifter (Seattle, WA, USA).

\section{Cell Culture}

Freshly isolated and negatively selected (RosetteSep) human B-cells, both from CLL patients and normal subjects, were cultured in IMDM media (Glutamax, GIBCO, Carlsbad, CA, USA) supplemented with $5 \%$ human $\mathrm{AB}$ serum (Atlanta Biologicals, Lawrenceville, GA, USA), 2 mM L-glutamine, $0.1 \mathrm{mM}$ 2-mercaptoethanol (Chemicon, Temecula, CA, USA), and penicillin (100 U/mL)streptomycin $(100 \mu \mathrm{g} / \mathrm{mL})$ (GIBCO-BRL Life Technologies; Grand Island, NY, USA). In addition, the following cell lines were used: MEC-1 (15); RAMOS (Burkitt's lymphoma, IgM); Dakiki (Epstein-Barr virus-transformed lymphoblast, IgA), Jurkat (acute T-cell leukemia); and 697 (acute lymphoblastic leukemia) (697 was obtained from DSMZ [Braunschweig, Germany]). 697-Bcl-2 cells were verified to have a 10 -fold higher expression of $\mathrm{Bcl}-2$ relative to $697-$ Neo cells (data not shown). 697 cells were maintained in RPMI 1640 supplemented with $10 \%$ human serum, plus glutamine and antibiotics. Other cell lines were maintained in RPMI medium (GIBCO) supplemented with $10 \%$ fetal bovine serum (FBS), penicillin (100 U/mL)streptomycin $(100 \mu \mathrm{g} / \mathrm{mL})$ (GIBCO-BRL), and $2 \mathrm{mM}$ L-glutamine (GIBCO-BRL). All cells were cultured in humidified air with $5 \% \mathrm{CO}_{2}$ at $37^{\circ} \mathrm{C}$. Cells were induced to undergo apoptosis by exposure to camptothecin (Sigma, St Louis, MO, USA) or by changes in culture temperature. In the latter case, cells were placed in an Eppendorf tube and immersed in a water bath or placed on a heat block for 3-5 min at either $45^{\circ}$ or $60^{\circ} \mathrm{C}$ before or after staining. Although either order yielded similar results, optimal data were obtained when cells were surface stained and then heated with a heat block. Flow cytometric gating was based on the isotype control antibody provided by the manufacturer and the amount of nonspecific signal in cells singly stained with Annexin V.

\section{Western Blot Analyses}

Cells were collected and washed with cold PBS, erythrocytes removed with lysis buffer containing protease inhibitors (both from Roche, Basel, Switzerland) and stored at $-20^{\circ} \mathrm{C}$. Next, $5 \mathrm{mM}$ ethylenediaminetetraacetic acid (EDTA), $150 \mathrm{mM} \mathrm{NaCl}, 0.1 \%$ sodium dodecyl sulfate (SDS), $0.1 \%$ sodium deoxycholate containing 1:40 dilution of protease inhibitor cocktail for mammalian cells and 1:50 dilution of phosphatase inhibitor cocktail 2 (both from Sigma-Aldrich, St. Louis, MO, USA) were added. The protein concentration of each cell lysate was determined with the RC and DC Protein Assay (Bio-Rad Laboratories, Hercules, CA, USA).

Proteins were separated by $10 \%$ SDS-polyacrylamide gel electrophoresis (PAGE) and transferred on Immobilon-P polyvinylidene difluoride membranes (Millipore, Bradford, MA, USA). Thirty $\mu \mathrm{g}$ of protein were loaded into each lane. 
Membranes were blotted at $4^{\circ} \mathrm{C}$ overnight with mouse anti-human $\mathrm{Bcl}-2$ (BD, Franklin Lakes, NJ, USA). Immunodetection was achieved with goat antimouse IgG horseradish peroxidase (HRP)-linked polyclonal antibodies (New England BioLabs, Ipswich, MA, USA) and the ECL Plus (Enhanced Chemiluminescence) detection system (Amersham Biosciences, Piscataway, NJ, USA) with BioMax MR films (Eastman Kodak, Rochester, NY, USA). Protein bands were analyzed by ImageQuant software (Amersham Biosciences).

\section{RT-QPCR}

The relative expression of $\mathrm{mRNA}$ was determined using the ABI PRISM 7700 Sequence Detection System and TaqMan chemistry (Applied Biosystems, Foster City, CA, USA). Briefly, a set of forward and reverse primers were designed with a probe labeled with a reporter at the 5 'end and a quencher at the $3^{\prime}$ end in an internal region of the target sequence. Fluorescence was directly proportional to the amount of target RNA. Fluorescent intensity was measured at every cycle, and cycle numbers were determined at a point in which every sample was in the exponential phase. All samples were analyzed in duplicate, and $\beta$-actin was used as an internal control gene. Results were obtained as Ct (Threshold cycle) values, in which Ct is inversely proportional to the starting template copy number. Relative expression of the target gene was calculated in comparison to untreated control samples using the delta delta Ct method (User Bulletin \#2; Applied Biosystems). Results were expressed as fold change with respect to the experimental control set being normalized to one. Primer sequences: Forward Primer: AAATCCATGCACCTAAACCTTTTG; Reverse: CAAATTCTACCTTGGAGGGA AAAAAC; Taqman Probe: CCGTG GGCCCTCCAGATAGCTCAT.

\section{Flow Cytometry}

Freshly isolated or thawed, negativelyselected B cells from B-CLL patients and normal healthy donors were washed with PBS containing $1 \%$ FBS and $0.1 \%$ sodium azide, and incubated for $20 \mathrm{~min}$ at $4^{\circ} \mathrm{C}$ with one of two mAbs (mouse 100 or hamster 6c8) specific for Bcl-2 (BD), with and without lineage specific antibodies to CD19, CD5, and CD11b. After fixation in $1 \%$ formaldehyde, cells were compared with others, similarly prepared but exposed to fluorochrome-conjugated isotype control mAbs, by flow sorting using a FACS-Aria (BD Biosciences, San Jose, CA, USA) or a FACS-Caliber (BD Biosciences). Cryopreserved samples from patients with acute lymphoblastic leukemia (ALL), hairy cell leukemia (HCL), and multiple myeloma (MM) were analyzed after costaining with $\mathrm{mAbs}$ specific for CD10, CD19, and CD138, respectively. Cells from untreated B-CLL patients and normal subjects were gated on the lymphocyte group prior to analysis; other untreated leukemias were gated on singlets. Experimental samples were analyzed as total cells or gated to eliminate debris.

Cell cycle changes and the occurrence of apoptosis were detected by flow cytometry after incubating cells with propidium iodide (PI; Calbiochem, San Diego, CA, USA). $1 \times 10^{6}$ cells were exposed to hypotonic PI $(0.05 \mathrm{mg} / \mathrm{mL}$ PI, $0.1 \%$ Triton $\mathrm{X}-100)$, and data were acquired on a FACS-Caliber with a 488-nm argon laser. In some instances, cells were examined for viability with Annexin V conjugated to FITC, PE, or APC (BD Biosciences). Acquisition and analysis were performed with CellQuest software (BD Biosciences) of FlowJo (Tree Star Inc, Ashland, OR, USA). Apoptotic cells in PI samples were calculated from the subG $\mathrm{G}_{1}$ fraction as previously described $(16,17)$.

\section{Confocal Microscopy}

Cells were washed and stained with appropriate $\mathrm{mAbs}$ as described for flow cytometry and then exposed to $4 \%$ paraformaldehyde for $20 \mathrm{~min}$, followed by another wash. Anti-fade reagent (ProLong Gold; V, Carlsbad, CA, USA) was added before mounting on slides with coverslips. Isotype control mAbs were used to evaluate non-specific binding. Images were collected using a $60 \times$ numerical aperture 1.4 PlanApo infinity-corrected objective with an Olympus Fluoview 300 confocal microscope (Olympus America Inc., Center Valley, PA, USA). Files were converted to bitmap in Paint (Microsoft, Redmond, WA, USA) and adjusted for brightness and contrast with Microsoft Picture Manager (Microsoft).

\section{Statistical Analyses}

Data were analyzed with Microsoft Excel for statistical significance using Student $t$ test. Two-tailed distributions were used except for experiments in which apoptosis was induced with camptothecin (Sigma, St Louis, MO, USA) or by heat, for which a one-tailed $t$ test was utilized, since these treatments would not be expected to increase viability. $P$ values $<0.05$ were considered statistically significant. Error bars represent standard deviations.

All supplementary materials are available online at molmed.org.

\section{RESULTS}

\section{$\mathrm{BCl}-2$ is Elevated in B-CLL at the RNA and Protein Levels}

B-CLL cells have enhanced levels of intracellular Bcl-2 (18), thereby assisting the tumor cells in evading elimination by apoptosis. We verified these findings, at the RNA level, by microarray analyses with two Affymetrix chips followed by RTQPCR, and, at the protein level, by intracellular immunofluorescence and Western blotting (Figure 1). Upon initial screening based on Affymetrix probe calls, 6 of 13 microarray probe sets showed highly significant increases in Bcl-2 mRNA levels. The average fold change for B-CLL patients $(n=35)$ compared with controls $(n=24)$ was 4.44 (Figure 1B), which correlated well with the average RT-QPCR fold change for 18 paired B-CLL and normal samples, 5.53 (Figure 1C).

Western blotting analyses comparing B-cell lysates of five B-CLL patients with those of three normal individuals matched for age also confirmed increased levels of Bcl-2 protein in all leukemic samples relative to control (Figure 1D). For the cohort of B-CLL samples 
A

\begin{tabular}{|c|c|c|}
\hline Chip & \multicolumn{1}{|c|}{ Fold } & T-Test \\
\hline U133 & 20.02 & $4.21 \mathrm{E}-06$ \\
\hline U133 & 14.19 & $5.46 \mathrm{E}-14$ \\
\hline U133 & 7.51 & $3.01 \mathrm{E}-05$ \\
\hline U95 & 3.88 & $3.14 \mathrm{E}-07$ \\
\hline U95 & 3.12 & $2.85 \mathrm{E}-05$ \\
\hline U95 & 2.22 & $4.59 \mathrm{E}-05$ \\
\hline
\end{tabular}

B

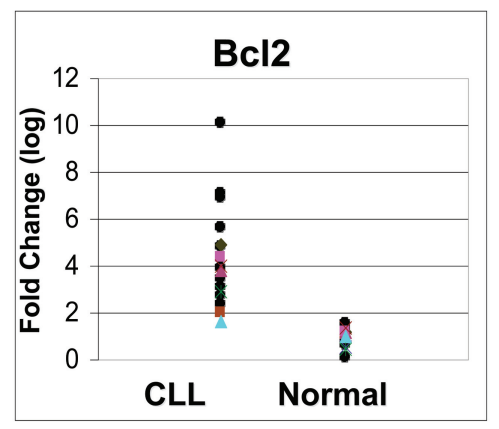

C

\begin{tabular}{|c|c|c|}
\hline QPCR & Fold & n \\
\hline Bcl2 & 5.53 & 36 \\
\hline
\end{tabular}

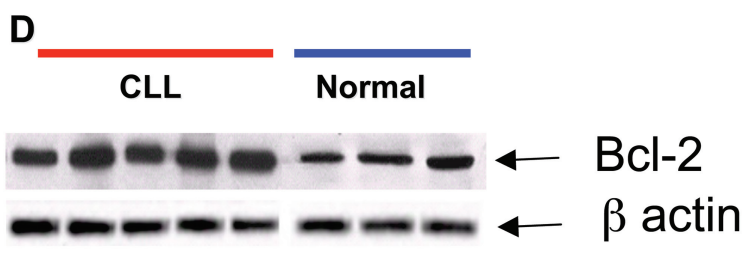

Figure 1. Expression of $\mathrm{BCl}-2 \mathrm{mRNA}$ and protein. (A) Microarray analysis of $\mathrm{BCl}-2$. Probe sets for $\mathrm{BCl}-2$ found to be significantly different at initial gene expression screening by microarray. The first column is the Affymetrix chip version, the second column is the fold change of B-CLL samples compared with samples from normal subjects $(n=59)$, and the third column is the t test value (one-tailed, type 2). (B) RT-QPCR verification of Bcl-2 levels. Graphic representation of data for the first round of RT-QPCR verification. Eighteen paired samples from B-CLL patients and normal individuals were analyzed for fold change differences. Control data were normalized to one and the delta delta CT method of analysis was employed. (C) Summary chart of the Bcl-2 RNA message. Numbers represent the mean log fold change of Bcl-2 mRNA as measured by RT-QPCR for the samples shown in Figure 1B. (D)Western blot analysis of Bcl-2 protein levels. Lysates of B cells from five B-CLL patients on the left and three healthy subjects matched for age on the right were separated by SDS-PAGE electrophoresis and probed with a Bcl-2-specific antibody. The presence of very similar amounts of $\beta$ actin in each lane indicates similar protein loading for each lane.

used in Figure 2, an average of $98.45 \%$ of cells expressed intracellular Bcl-2 by immunofluorescence (data not shown).

\section{$\mathrm{BCl}-2$ Detected on the Surface Membrane of B-CLL Cells}

We exposed freshly drawn, never frozen B cells from U-CLL $(n=19)$ and M-CLL $(n=20)$ patients to FITC-labeled mAbs specific for Bcl-2 (see Figure 2, Supplemental Figure 1) and analyzed for surface membrane (sm) expression of the protein by flow cytometry.

Leukemic B cells clearly expressed smBcl-2 (Figure 2A). This was the case for both M-CLL and U-CLL clones, and there was no statistically significant difference in smBcl-2 levels between the two patient subgroups $(P=0.65)$. SmBcl-2 expression by B-CLL cells was verified with the same specific $\mathrm{mAb}$ linked to a different fluorochrome, PE; furthermore, analyses with another $\mathrm{mAb}$ clone isolated from a different species, Armenian hamster, yielded similar results (data not shown).

The pattern of smBcl-2 expression varied among B-CLL clones, ranging from distinct, dense populations of $\mathrm{CD} 19^{+} \mathrm{CD}^{+}{ }^{+} \mathrm{smBcl}-2^{+}$cells (Figure $2 \mathrm{~B}$, far right column) to $\mathrm{CD} 19^{+} \mathrm{CD}^{+}{ }^{\mathrm{smBcl}-2^{+}}$ populations containing fewer cells (Figure $2 \mathrm{~B}$, second column from right). Finally, a subset of clones expressed only a few $\mathrm{CD} 19^{+} \mathrm{CD}^{+}{ }^{\mathrm{smBcl}}-2^{+}$cells (Figure 2B, third column from right).

\section{Bcl-2 Detected on the Surface Membrane of Normal B Lymphocytes but Not B-Lineage Cell Lines}

We next evaluated smBcl-2 expression on fresh human B lymphocytes from healthy, age-matched subjects. Normal B cells expressed smBcl-2, albeit at levels less than that of B-CLL cells (Figure 2A, 2B). This difference in membrane expression was highly significant when compared with B-CLL as a whole $\left(P=3.75 \times 10^{-6}\right.$; see Figure $2 \mathrm{~A}$ ) or with its subtypes (U-CLL, $P=$ $8.26 \times 10^{-5}$ and M-CLL, $P=5.05 \times 10^{-6}$ ).

In addition, we analyzed smBcl-2 expression on human cord blood B cells. These cells were chosen because of surface membrane expression of CD5, a characteristic marker for CLL $(19,20)$. Cord blood expression of smBcl-2 was not of the same magnitude as seen in B-CLL, as evidenced by the insignificant $P$ value for the $\mathrm{CD}^{+}(P=0.07)$ as well as $\mathrm{CD5}^{-}(P=$ 0.21 ) cord blood cells. Although smBcl-2 expression was more evident among $\mathrm{CD}^{+}$cord blood cells $(n=5)$ than CD5 cord blood cells $(n=7)$ from the same samples (2.39\% - 5.07\%; see Figure $2 \mathrm{~A})$, this difference was not significant.

Several cell lines also were analyzed. Dakiki, an EBV transformed, IgAexpressing B-cell line from a patient with nasopharyngeal carcinoma; Jurkat, a $\mathrm{T}$ lymphoblast cell line from a patient with acute T cell leukemia; and MEC1, a transformed B-cell line derived from a B-CLL patient, were each negative for smBcl-2 (data not shown). Similarly, a human acute lymphoblastic leukemia cell line, 697, which was transfected with $\mathrm{Bcl}-2$, did not have detectable smBcl-2, before or after transfection. RAMOS B cells, a Burkitt's lymphoma cell line, showed some positive cells, particularly after apoptotic induction by irradiation, although a large amount of non-specific binding occurred after apoptosis induction (data not shown).

\section{Relationship between smBcl-2 and Cell Viability in B-CLL and Normal Human B Cells}

Because mouse B cells can express smBcl-2 during apoptosis (21), we ex- 
A

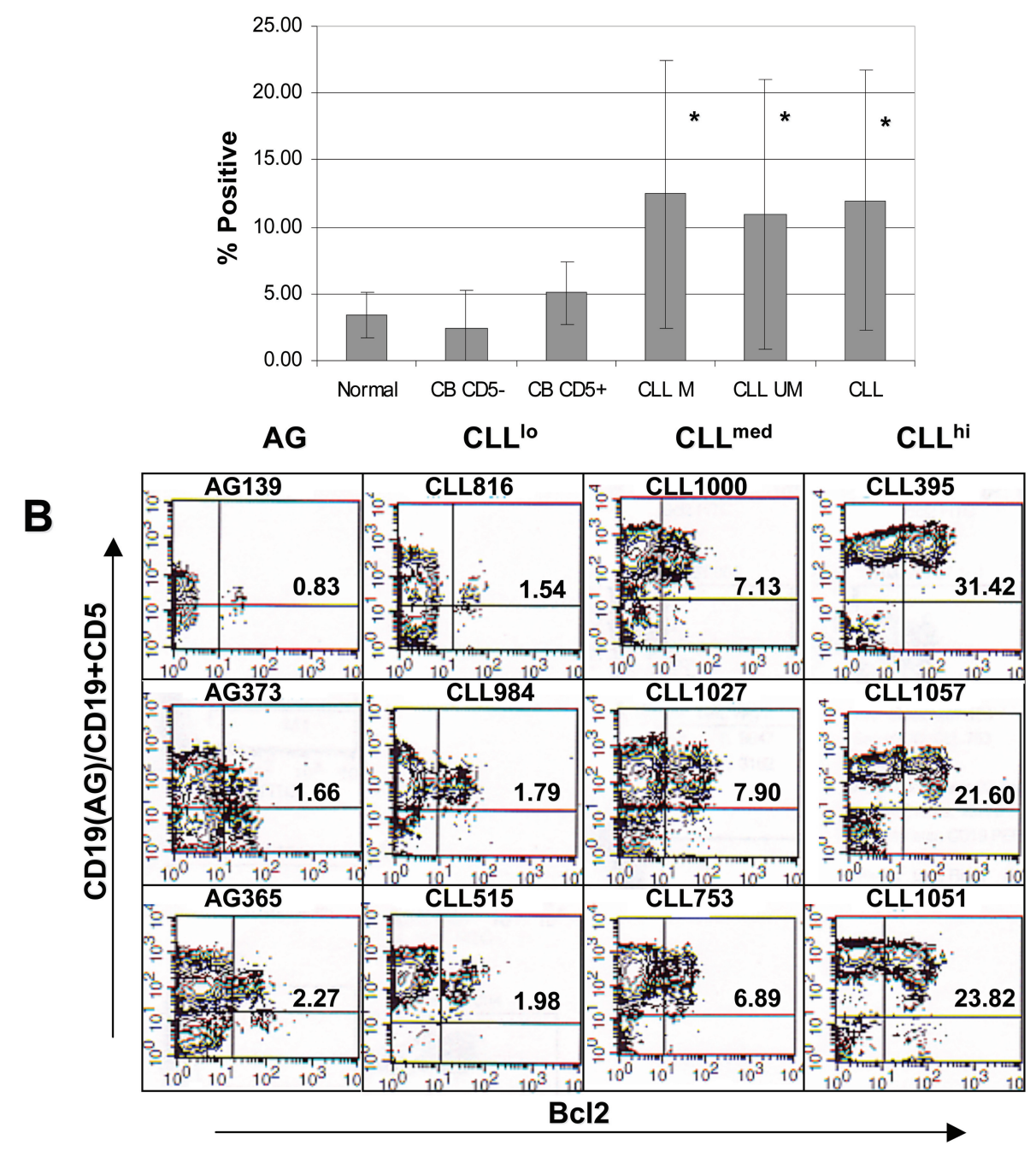

Figure 2. Flow cytometric analysis of $\mathrm{Bcl}-2$ surface membrane expression. (A) Bcl-2 levels on fresh B cells. Graphic representation of the percent of $\mathrm{smBCl}-2^{+}$cells in $\mathrm{B}$ cells isolated from age-matched normal individuals, $\mathrm{CD}^{-}$and $\mathrm{CD}^{+}$fractions enriched from cord blood B cells, M-CLL and U-CLL patients, and a composite of the B-CLL patients were compared after flow cytometric staining with the PE-conjugated mouse anti-human $\mathrm{BCl}-2$ $\mathrm{mAb}$ 100. (B) Population analysis of smBcl-2 $2^{+}$cells. Contour plot of $\mathrm{Bcl}-2$ levels in $\mathrm{B}$ cells from age-matched healthy controls and B-CLL patients. Normal controls are pictured as CD19 (y-axis) by BCl2 (x-axis). B-CLL samples are first gated on CD19 and then shown as CD5 (y-axis) by Bcl2 (x-axis).

amined smBcl-2 expression on human B lymphocytes from B-CLL patients and normal subjects undergoing cell death. First, thawed cells, which would be expected to exhibit increased levels of apoptosis over fresh cells, were analyzed. Comparing these data to those from cells that had not been cryopreserved, the average percent $\mathrm{smBcl}-2^{+}$ cells in frozen/thawed samples was increased significantly compared with cells that had never been frozen and thawed (11.99 versus 23.41, respectively, $P=0.01$; Figure 3A). Similar to fresh cells (see Figure 2), there was a significant difference in smBcl-2 levels between B-CLL cells and B cells from normal subjects $(P=0.03$; see Figure $3 \mathrm{~A})$. However, there was no significant difference between M-CLL or U-CLL and normal controls in the thawed subset, possibly because of sample size ( $P=$ 0.056 and 0.16 , respectively).

Next, lymphoid populations from B-CLL patients were examined by flow cytometry, using forward scatter to divide the populations into smaller cells (R1), typically increased among dying/ dead cells, and larger (R2), more viable cells. smBcl-2 expression was markedly enriched in the smaller, dying/dead cell population than in larger, healthier cells. For patient 435 , virtually all smBcl-2 was found on small cells, while, for patient 753, a lesser percentage, but still mostly the small cells, were smBcl- $2^{+}$ (Figure 3B).

To correlate smBcl-2 expression with the occurrence of apoptosis specifically, we analyzed freshly isolated B cells of both B-CLL and normal subjects by flow cytometry for smBcl-2 and cell viability, using either PI or Annexin V approaches. Consistent with other data sets, there was a significant difference in smBcl- $2^{+}$ cells when comparing B-CLL and normal B cells ( $P=0.02$; Figure 4A, Supplemental Figure 2). The numbers of smBcl-2 ${ }^{+}$ and Annexin $\mathrm{V}^{+}$cells were elevated in B-CLL compared with normal controls, suggesting that, as apoptosis proceeded, smBcl-2 expression increased.

Finally, to directly examine the appearance of smBcl-2 during the apoptotic process, B cells were analyzed after inducing apoptosis by multiple methods: exposure to non-physiologic temperatures: $4^{\circ} \mathrm{C}$ (data not shown), $45^{\circ} \mathrm{C}$ (data not shown), and $60^{\circ} \mathrm{C}$ (Figure $4 \mathrm{~B}$, Supplemental Data Figure 3); or by exposure to camptothecin, a chemical that induces apoptosis in B-CLL cells (22) by affecting expression of Bcl-2 family members (23) (Figure 5A, Supplemental Data Figure 4). Figure 4B is representative of repeated experiments heating normal B-cells at $60^{\circ} \mathrm{C}$ for $3 \mathrm{~min}$. Expos- 
A

Surface membrane Bcl-2

(Frozen and Thawed Cells)

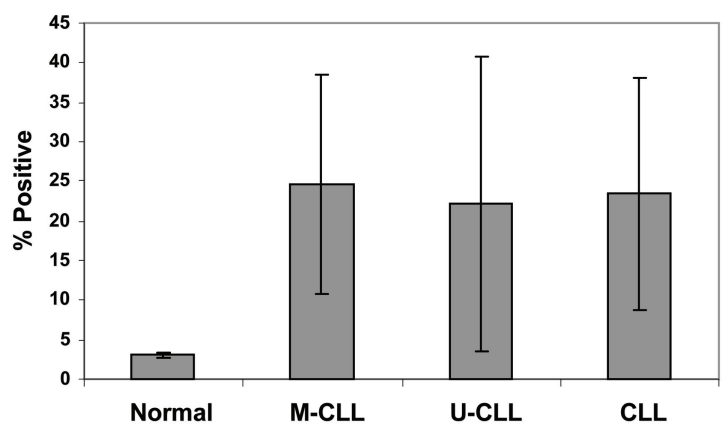

B
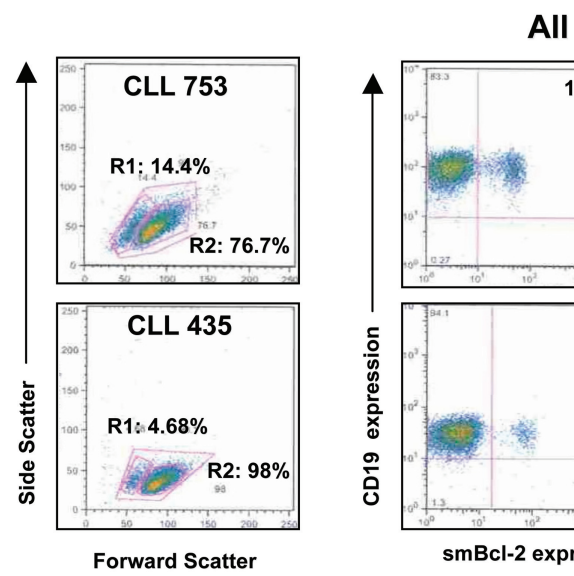

R2

R1

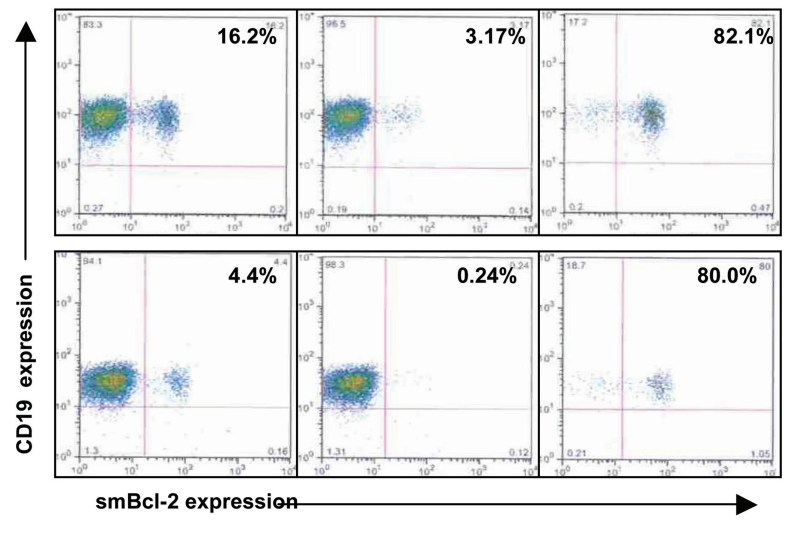

Figure 3. Patterns of smBcl-2 expression. (A) Analysis of smBcl-2 levels on frozen cells. Graphic representation of $\mathrm{BCl}-2$ levels in $\mathrm{B}$ cells from age-matched healthy subjects (first bar on left), M-CLL patients (second bar), and all B-CLL patients combined (right bar). Data were analyzed with Student $t$ test. A significant difference was found between B-CLL patients and normal control subjects $(P<0.03)$. (B) Analysis of gated cell populations. Two B-CLL samples were analyzed after gating into subpopulations based on forward and side scatter (left column), ungated (second column) R2 cells only (third column), or R1 cells only (fourth column). Numbers represent the R1 and R2 populations in the left graph and percent double positive cells, CD19 (y-axis) and Bcl2 (x-axis), in the remaining.

ing normal B cells $(n=9)$ to these conditions always induced significant levels of apoptosis, but not always a significant increase in smBcl-2 expression. The corresponding $t$ tests for cells heated 3 min (Supplemental Figure 3A,3B) are shown in Supplemental Data Figure 3D. Cells exposed to $60^{\circ} \mathrm{C}$ for $4 \mathrm{~min}$ induced over $98 \%$ Annexin V-binding cells with an increase in smBcl-2 of $\sim 33 \%$. Since the degree of smBcl-2 expression was not always proportional to the level of apoptosis induction at 3 or 4 min, it is difficult to draw a clear conclusion as to the relevance of smBcl-2. Of note, treating B-CLL cells with $10 \mu \mathrm{M}$ camptothecin for $1 \mathrm{~d}$ significantly induced apoptosis in both B-CLL cells and normal aging $B$ cells $(P=0.01$; Figure 5A); however only B-CLL cells expressed significantly more smBcl-2 $(P=$ 0.03; Figure 5A). Figure 5B illustrates an example of enhanced smBcl-2 expression by Annexin $V^{\text {high }}$ versus Annexin $\mathrm{V}^{\text {low }}$ members of a B-CLL clone (note scale difference).

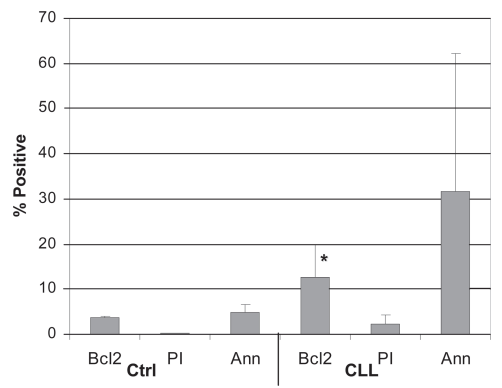

B

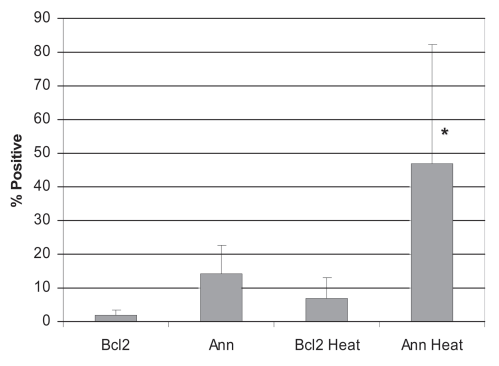

Figure 4. Correlation between $\mathrm{smBcl}-2$ expression and induction of apoptosis. (A) Analysis of fresh cells. B-cells from B-CLL patients and age-matched normal controls, that were never frozen, were analyzed simultaneously for smBcl-2 expression (left bar) and for apoptosis $(n=12)$. PI was used to quantify apoptosis by DNA fragmentation (middle bar) $(n=6)$ as well as Annexin $V$ positivity (right bar) $(n=6)$. Asterisk indicates a significant difference in levels of smBcl-2 between normal and $\mathrm{B}-\mathrm{CLL}$ samples $(P=0.02)$. (B) Heat-challenged normal B cells. Normal $B$ cells from normal age-matched subjects were exposed to a $60^{\circ} \mathrm{C}$ hot water bath and then analyzed for smBcl-2 and viability by Annexin $V$ binding; levels are shown before heating (left) and after (right), respectively.

\section{Microscopic Examination of smBcl-2 Expression}

To further corroborate surface membrane expression of Bcl-2, confocal microscopy was performed (Figure 6). Similar to results obtained with flow cytometry (see Figure 2), heterogeneous patterns of smBcl-2 expression were observed, and some B-CLL clones contained more smBcl-2+ cells (Figure 6A) than others (Figure 6B,6C). 
A.

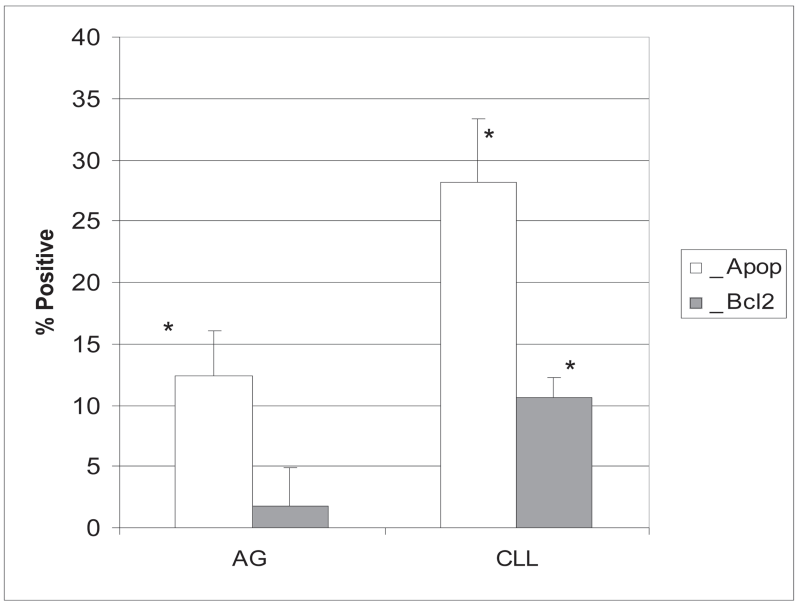

B.

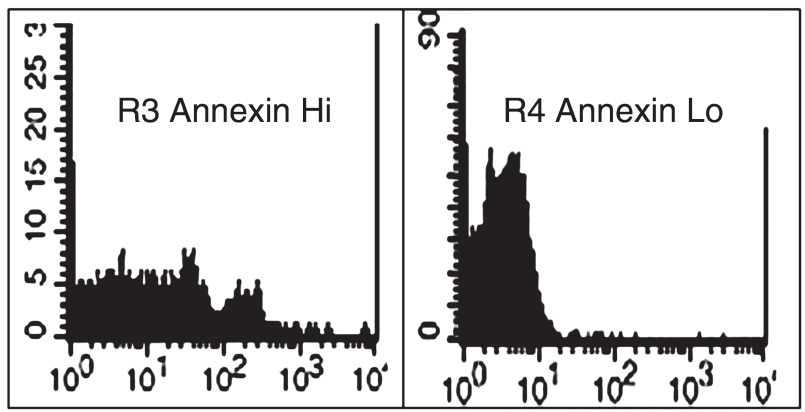

Figure 5. Levels of smBcl-2 after chemical induction of apoptosis. (A) Apoptosis induction by camptothecin. Graphic representation of the differences in levels of apoptosis (white bar) and smBcl-2 (gray bar) after camptothecin treatment. Cells from normal, age-matched (AG) control B cells (left) and B-CLL patients (right) were exposed to $10 \mu \mathrm{M}$ camptothecin for $1 \mathrm{~d}$ and then analyzed for $\mathrm{smBcl}-2$ and apoptosis. Apoptosis was induced significantly in both groups. The change in smBcl-2 was significant only for $\mathrm{B}-\mathrm{CLL}$ cells $(P=0.03)$. (B) Annexin and smBcl-2 co-expression on CLL cells. Histogram of a representative B-CLL sample gated on cells with high (left) or low (right) levels of Annexin $\vee$ binding.

\section{smBcl-2 Detected in Other B-cell Malignancies Lacking $t(14 ; 18)$}

To determine if smBcl-2 expression was unique to B-CLL, cells from other B-cell leukemias and lymphoproliferative disorders were analyzed. smBcl-2 was detected on B cells from patients with ALL (Figure 7A-D), HCL (Figure 7E), and MM (Figure 7F). Of note, smBcl-2 was found at very high levels in virtually every ALL sample analyzed. Rows A-C illustrate data with unsorted samples, while rows D-F are data generated from cells sorted as described in Materials and Methods. The differences in ALL3 between rows $\mathrm{A}$ and $\mathrm{D}$ may represent cell debris generated by cell sorting. In addition, a difference was seen between two HCL cases studied (225 and 1317): levels of smBcl-2 ${ }^{+}$cells were similar ( $85.8 \%$ and $89.5 \%$, respectively), although the subG1 apoptotic peak dominated for HCL225 but was only a minor population for HCL1317; thus, in this latter instance, more smBcl-2 was detected among viable cells.

We also confirmed that smBcl-2 expression was enriched among dying cells from these other B-cell derived neoplasms, using simultaneous flow cytometry for smBcl-2 expression and PI analysis for DNA fragmentation (Figure 7D-F). These studies showed a clear correlation of smBcl-2 levels with the numbers of cells undergoing apoptosis in each disease.

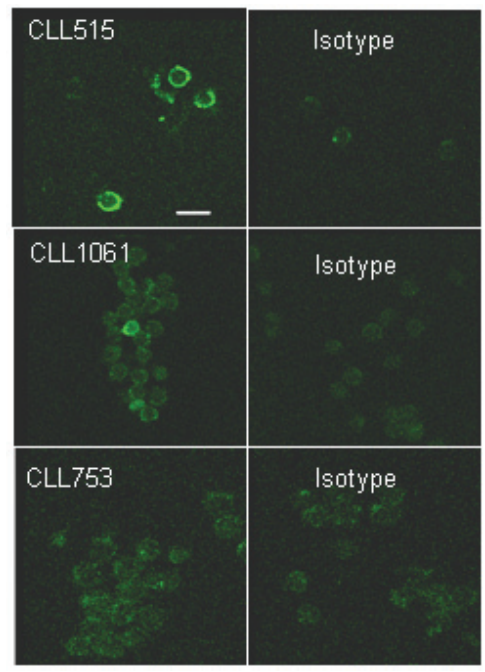

Figure 6. Confocal microscopy reveals $\mathrm{BCl}-2$ on the surface of B-CLL cells. Fresh, never frozen, B-CLL cells are shown as representative of at least triplicate microscope fields, surface stained for Bcl-2 FITC (mAb 100, left panel) and an isotype control antibody (right panel). The bar scale (in the top left picture) indicates $10 \mathrm{mM}$.

\section{DISCUSSION}

In this study, we confirmed elevated Bcl-2 levels within B-CLL cells at the RNA and protein level. Microarray results from two different Affymetrix chips, U95 and U133, were verified by RT-QPCR and Western blot analyses (see Figure 1). We also uncovered the surprising presence of $\mathrm{Bcl}-2$ on the surface of leukemic B cells, identified by flow cytometry (see Figure 2), and corroborated by confocal microscopy (see Figure 6). Although variable on a case-by-case basis, B-CLL cells had significantly increased levels of smBcl-2 (see Figure 2) on both fresh and frozen cells when compared with B cells from the blood of normal neonates and adult subjects.

smBcl-2 expression has been reported in the murine thymocyte cell line, EL-4 (21), and its presence detected in the membrane fractions of cell lines and lymphomas with the $t(14 ; 18)$ translocation (24). Chen-Levy visualized low levels of smBcl-2 by phase contrast microscopy in a human follicular lymphoma cell line that carries the 


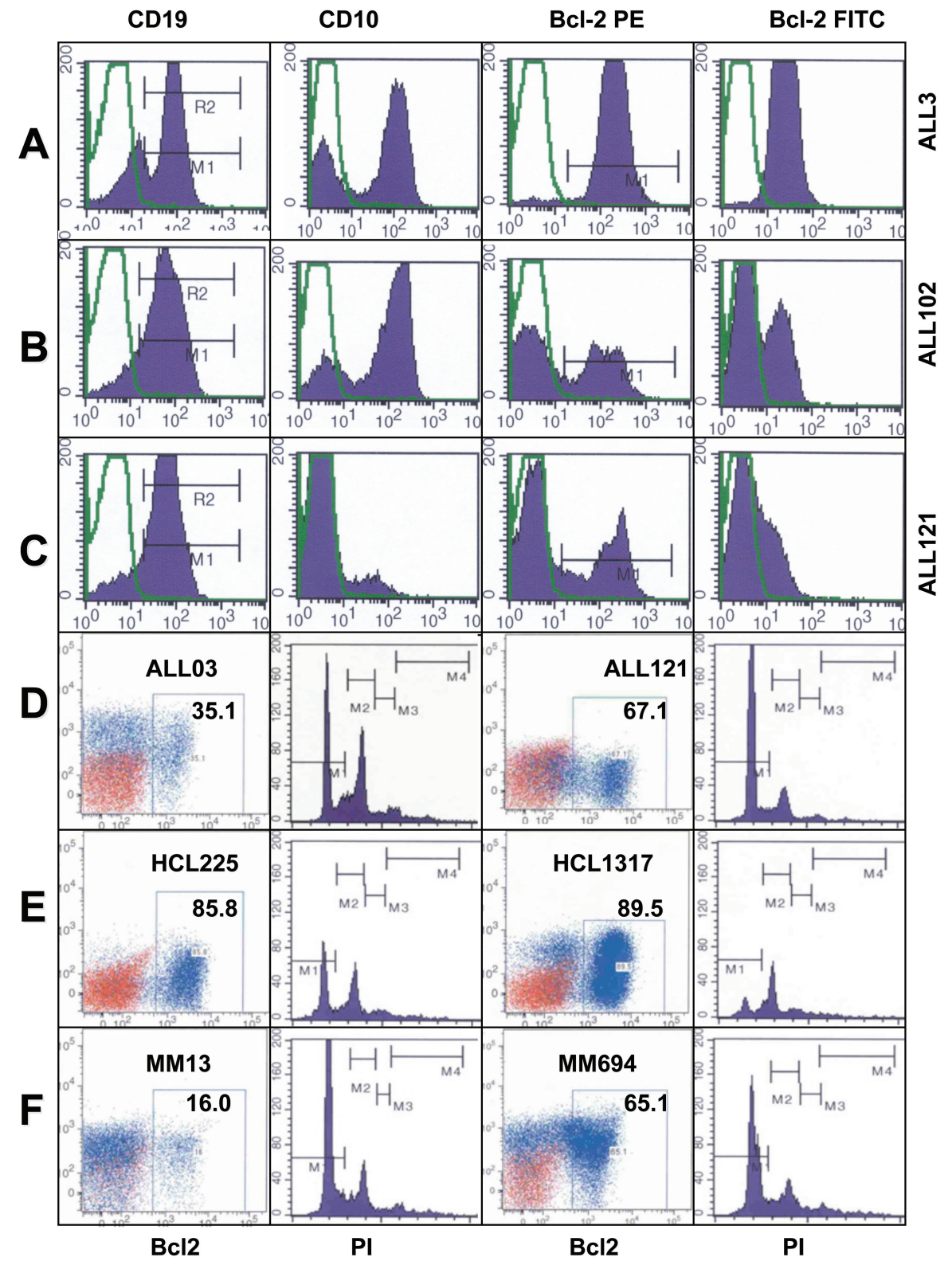

Figure 7. smBcl-2 expression in other leukemias. (A-C) smBcl-2 expression in acute lymphocytic leukemia (ALL). Three ALL samples were stained for CD19 (column 1), CD10 (characteristic of ALL; column 2), PE mouse anti-human BCl-2 (column 3), and FITC mouse antihuman BCl-2 (column 4). (D-F) smBcl-2 expression in non-CLL lymphoproliferative disorders. ALL (D), HCL (E), and MM (F) samples were FACS sorted for expression of CD19 or a more specific characteristic marker (see Materials and Methods), and then analyzed for smBcl-2 expression and apoptosis by PI. Numbers represent the percentages of smBcl- $2^{+}$cells.

$t(14 ; 18)$ translocation. In a cell line retrovirally transfected to overexpress Bcl-2, the plasma membrane fraction contained $\mathrm{Bcl}-2$ protein, while control cells did not (25), leading to the proposal that Bcl-2 localized to plasma membranes sup- pressed oxidative damage, as it does for mitochondrial membranes, and thereby had functional significance, possibly providing a survival advantage to these cells. Similarly, when Bcl-2 was observed in the membrane of mouse cells under- going apoptosis, it was proposed that Bcl-2, a known antioxidant (26-28), might maintain plasma membrane integrity as it does at the mitochondrial membrane.

Although smBcl-2 was enhanced in dying cells, in several cases apparently healthy cells were smBcl- $2^{+}$, and, therefore, we cannot rule out a function for smBcl-2 in viable cells. Therefore, our finding of smBcl-2 occurring at lower levels on adult and cord blood B cell populations than on B-CLL cells suggests exaggeration of a normal function in leukemic clones. However, using surface markers to identify cellular health can be misleading at times. For example, smAnnexin A1 on HCL cells might lead to the false conclusion that these were compromised cells. In fact, Annexin A1 binding to the cell surface is seen with such consistency that it is now a marker for the disease (29). In this study, HCL cases exhibited a large percent of $\mathrm{smBcl}-2^{+}$cells, as did previously cryopreserved ALL samples.

In examining the functional relevance of smBcl-2 and its upregulation during apoptosis, it may be asked whether cells positive for smBcl-2 are utilizing the molecule in a functional manner or if the appearance of the protein in the plasma membrane is merely a reflection of altered lipid bilayer integrity. We currently do not have data supporting or excluding either possibility. Several rescue points have been identified during apoptosis $(30,31)$. However, since smBcl-2 levels are increased in the small, probably dying, cell population identified by forward and side scatter (see Figure 3B) and by enhanced expression of Annexin V (see Figure 5B), but are not associated with DNA fragmentation defined by PI analysis (see Figure 4A), a mid-point during the apoptotic process is suggested as a targeted checkpoint by our studies. Takashina et al. have described rescue of HEK293 cells from apoptosis after activation of caspase 3 and appearance of membrane blebbing (32). This stage was associated with absent-to-low levels of Annexin V binding. Although the mech- 
anism responsible for the rescue of cells displaying membrane blebbing was not defined, upregulation of Bcl-2, along with cFLIP and IAP, did not occur. However, it should be mentioned that translocation of existing Bcl-2 to the plasma membrane was not considered. Although these findings are not consistent with prior findings indicating a checkpoint that precedes membrane blebbing $(4,5)$, reversal of membrane blebbing also has been reported for pancreatic acinar cells over-stimulated by specific agonists (33). Although cancer cells presumably have many mechanisms to attempt to avoid cell death (for example, epigenetic silencing of DAPK1, a protein thought to assist in early apoptosis through promoter methylation that has been observed in almost all cases of sporadic B-CLL analyzed, (34), our data leave the possibility open that smBcl-2 may provide an additional advantage for the B-CLL clone during apoptosis. This may not be unreasonable considering the documentation that $\mathrm{Bcl}-2$ and other family members become ER membrane-associated with stress (for example, excessive amounts of folded proteins [35], leading to survival activities such as autophagy [36]). In addition, the contribution of the Bcl-2 family to other activities not associated with apoptosis needs to be considered, that is, involvement in the cell cycle (37), repair of damaged DNA $(37,38)$, and metabolism of glucose (39).

In summary, the presence of $\mathrm{Bcl}-2$ on the surface membrane of B-CLL cells and B cells of certain other lymphoproliferative disorders is a surprising finding, although, at this juncture, it is not clear if smBcl-2 expression is a passive consequence of the apoptotic process or an active attempt to abort cell death by stabilizing the surface membrane. Further investigations of the functional relevance of this finding are warranted.

\section{ACKNOWLEDGMENTS}

The authors thank Herb Borerro, Tom McCloskey, Stella Stefanova, and Amanda Chan of The Feinstein Institute, Manhasset, New York, United States of
America, and Carolyn Cheney of Ohio State University, Columbus, Ohio, United States of America, for their help with flow cytometric and confocal microscopic analyses. The Bcl-2-overexpressing variant of 697,697-Bcl-2, and its empty-vector control, 697-Neo22, were the kind gift of Drs. Shinichi Kitada and John Reed of the Burnham Institute for Medical Research, La Jolla, California, United States of America. We also are grateful to N Muthusamy, Ohio State University, for assistance with the 697 cell experiments, and to Gloria Telusma, Rahena Chowdhury, and Somara Rafiq for assistance with B cell isolation. Finally, we acknowledge the insights of Drs. Patricia Mongini, Michael Grever, and John Byrd in preparation of the manuscript.

These studies were supported in part by RO1 grant CA 87956 from the NIH National Cancer Institute, M01 General Clinical Research Center Grant (RR018535) from the NIH National Center for Research Resources, and fellowship grants from the Lauri Strauss Leukemia Foundation. The Karches Foundation, Peter Jay Sharp Foundation, Prince Family Foundation, Marks Foundation, the Jean Walton Fund for Lymphoma and Myeloma Research, and the Joseph Eletto Leukemia Research Fund also provided support for these studies.

\section{REFERENCES}

1. Tsujimoto $Y$, Gorham J, Cossman J, Jaffe E, Croce CM. (1985) The $t(14 ; 18)$ chromosome translocations involved in B-cell neoplasms result from mistakes in VDJ joining. Science 229:1390-3.

2. Reed JC. (2008) Bcl-2-family proteins and hematologic malignancies: history and future prospects. Blood 111:3322-30.

3. Chao DT, Korsmeyer SJ. (1998) BCL-2 family: regulators of cell death. Annu. Rev. Immunol. 16:395-419.

4. Danial NN, Korsmeyer SJ. (2004) Cell death: critical control points. Cell 116:205-19.

5. Danial NN. (2007) BCL-2 family proteins: critical checkpoints of apoptotic cell death. Clin. Cancer Res. 13:7254-63.

6. Ciardiello F, Tortora G. (2002) Inhibition of bcl-2 as cancer therapy. Ann. Oncol. 13:501-2.

7. Del Gaizo Moore V, et al. (2007) Chronic lymphocytic leukemia requires BCL2 to sequester prodeath BIM, explaining sensitivity to BCL2 an- tagonist ABT-737. J. Clin. Invest. 117:112-21.

8. Shirane M, Nakayama KI. (2003) Inherent calcineurin inhibitor FKBP38 targets Bcl-2 to mitochondria and inhibits apoptosis. Nat. Cell. Biol. 5:28-37.

9. Fischer G, Aumuller T. (2003) Regulation of peptide bond cis/trans isomerization by enzyme catalysis and its implication in physiological processes. Rev. Physiol. Biochem. Pharmacol. 148:105-50.

10. Nguyen M, Millar DG, Yong VW, Korsmeyer SJ, Shore GC. (1993) Targeting of Bcl-2 to the mitochondrial outer membrane by a $\mathrm{COOH}$-terminal signal anchor sequence. J. Biol. Chem. 268:25265-8.

11. Yang J, et al. (1997) Prevention of apoptosis by Bcl-2: release of cytochrome c from mitochondria blocked. Science 275:1129-32.

12. Green DR, Reed JC. (1998) Mitochondria and apoptosis. Science 281:1309-12.

13. Krajewski S, et al. (1993) Investigation of the subcellular distribution of the bcl-2 oncoprotein: residence in the nuclear envelope, endoplasmic reticulum, and outer mitochondrial membranes. Cancer Res. 53:4701-14.

14. Portier BP, Taglialatela G. (2006) Bcl-2 localized at the nuclear compartment induces apoptosis after transient overexpression. J. Biol. Chem. 281:40493-502.

15. Stacchini A, et al. (1999) MEC1 and MEC2: two new cell lines derived from B-chronic lymphocytic leukaemia in prolymphocytoid transformation. Leuk. Res. 23:127-36.

16. Nicoletti I, Migliorati G, Pagliacci MC, Grignani F, Riccardi C. (1991) A rapid and simple method for measuring thymocyte apoptosis by propidium iodide staining and flow cytometry. J. Immunol. Methods 139:271-9.

17. McCloskey TW, Oyaizu N, Coronesi M, Pahwa S. (1994) Use of a flow cytometric assay to quantitate apoptosis in human lymphocytes. Clin. Immunol. Immunopathol. 71:14-8.

18. Gottardi D, et al. (1996) In leukaemic CD5+ B cells the expression of BCL-2 gene family is shifted toward protection from apoptosis. $\mathrm{Br}$. J. Haematol. 94:612-8.

19. Bhat NM, et al. (1992) The ontogeny and functional characteristics of human B-1 (CD5+ B) cells. Int. Immunol. 4:243-52.

20. Kipps TJ. (1989) The CD5 B cell. Adv. Immunol. 47:117-85.

21. Sapozhnikov AM, Gusarova GA, Ponomarev ED, Telford WG. (2002) Translocation of cytoplasmic HSP70 onto the surface of EL-4 cells during apoptosis. Cell. Prolif. 35:193-206.

22. Silber R, et al. (1994) Chemosensitivity of lymphocytes from patients with B-cell chronic lymphocytic leukemia to chlorambucil, fludarabine, and camptothecin analogs. Blood 84:3440-6.

23. Mei Y, et al. (2007) Noxa/Mcl-1 balance regulates susceptibility of cells to camptothecin-induced apoptosis. Neoplasia 9:871-81.

24. Chen-Levy Z, Nourse J, Cleary ML. (1989) The bcl-2 candidate proto-oncogene product is a $24-$ 
kilodalton integral-membrane protein highly expressed in lymphoid cell lines and lymphomas carrying the $\mathrm{t}(14 ; 18)$ translocation. Mol. Cell. Biol. 9:701-10.

25. Bruce-Keller AJ, et al. (1998) Bcl-2 protects isolated plasma and mitochondrial membranes against lipid peroxidation induced by hydrogen peroxide and amyloid beta-peptide. J. Neurochem. 70:31-9.

26. Steller H. (1995) Mechanisms and genes of cellular suicide. Science 267:1445-9.

27. Hockenbery DM, Oltvai ZN, Yin XM, Milliman CL, Korsmeyer SJ. (1993) Bcl-2 functions in an antioxidant pathway to prevent apoptosis. Cell 75:241-51.

28. Nguyen M, et al. (1994) Role of membrane anchor domain of Bcl-2 in suppression of apoptosis caused by E1B-defective adenovirus. J. Biol. Chem. 269:16521-4.

29. Falini B, et al. (2004) Simple diagnostic assay for hairy cell leukaemia by immunocytochemical detection of annexin A1 (ANXA1). Lancet 363:1869-70.

30. Ellis RE, Yuan JY, Horvitz HR. (1991) Mechanisms and functions of cell death. Annu. Rev. Cell. Biol. 7:663-98.

31. Hacker G, Vaux DL. (1997) A chronology of cell death. Apoptosis 2:247-56.

32. Takashina T, Nakayama M. (2007) Revival of apoptotic cells that display early-stage dynamic membrane blebbing. FEBS Lett. 581:4479-84.

33. Torgerson RR, McNiven MA. (1998) The actinmyosin cytoskeleton mediates reversible agonistinduced membrane blebbing. J. Cell. Sci. 111 (Pt 19): 2911-22.

34. Raval A, et al. (2007) Downregulation of deathassociated protein kinase 1 (DAPK1) in chronic lymphocytic leukemia. Cell 129:879.

35. Schroder M, Kaufman RJ. (2005) The mammalian unfolded protein response. Annu. Rev. Biochem. 74:739-789.

36. Maiuri MC, Zalckvar E, Kimchi A, Kroemer G. (2007) Self-eating and self-killing: crosstalk between autophagy and apoptosis. Nat. Rev. Mol. Cell. Biol. 8:741-52.

37. Zinkel S, Gross A, Yang E. (2006) BCL2 family in DNA damage and cell cycle control. Cell. Death Differ. 13:1351-9.

38. Kamer I, et al. (2005) Proapoptotic BID is an ATM effector in the DNA-damage response. Cell 122:593-603.

39. Danial NN, et al. (2003) BAD and glucokinase reside in a mitochondrial complex that integrates glycolysis and apoptosis. Nature 424:952-6. 